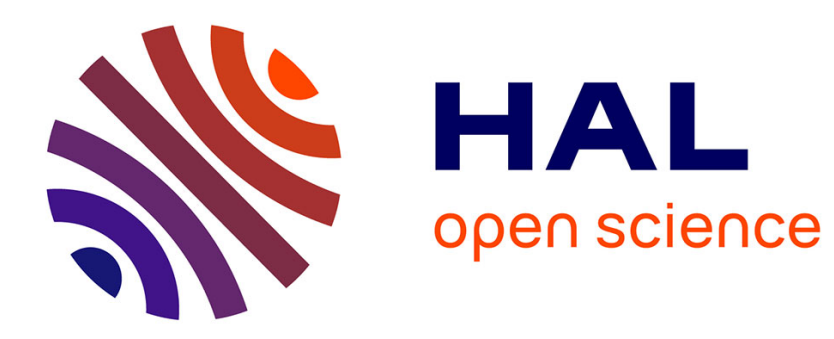

\title{
AC response of Abrikosov vortices in layered superconductors
}

S. Artemenko, C. Krämer, W. Wonneberger

\section{To cite this version:}

S. Artemenko, C. Krämer, W. Wonneberger. AC response of Abrikosov vortices in layered superconductors. Journal de Physique IV Proceedings, 1993, 03 (C2), pp.C2-79-C2-82. 10.1051/jp4:1993216 . jpa-00251585

\section{HAL Id: jpa-00251585 https://hal.science/jpa-00251585}

Submitted on 1 Jan 1993

HAL is a multi-disciplinary open access archive for the deposit and dissemination of scientific research documents, whether they are published or not. The documents may come from teaching and research institutions in France or abroad, or from public or private research centers.
L'archive ouverte pluridisciplinaire HAL, est destinée au dépôt et à la diffusion de documents scientifiques de niveau recherche, publiés ou non, émanant des établissements d'enseignement et de recherche français ou étrangers, des laboratoires publics ou privés. 


\title{
AC response of Abrikosov vortices in layered superconductors
}

\author{
S.N. ARTEMENKO, C. KRÄMER* and W. WONNEBERGER* \\ Institute of Radioengineering \& Electronics of the Russian Academy of Sciences, Mokhovaya 11, \\ 103907 Moscow, Russia \\ ${ }^{*}$ Department of Physics, University of Ulm, 7900 Ulm, Germany
}

\begin{abstract}
In a layered high- $T_{c}$ superconductor an Abrikosov vortex line perpendicular to the layers can be considered as a stack of coupled two-dimensional "pancake" vortices (2DV) with cores residing inside the superconducting layers. When the in-plane penetration length $\lambda_{a b}=\lambda$ is smaller than the Josephson length $\lambda_{J}=\lambda_{c} d / \lambda$ (d: layer period) the interaction between 2DV is via a magnetic pair force which is complicated but exactly known. We calculate linear and nonlinear ac responses for this model including randomly distributed pinning centers. In detail, the thermally averaged shift $S_{n}(t)$ of a $2 \mathrm{DV}$ in layer $n$ under the influence of a finite external ac current in the frequency range up to $1 \mathrm{GHz}$ is computed from coupled Fokker-Planck equations for the distribution functions of 2DV positions. In the adiabatic approximation, the Fokker-Planck equations can be cast into a nonlinear differential equation for a continuous shift function $S(z, t)$ using a gradient expansion. The constitutive equation for $S(z, t)$ is solved perturbatively for the linear ac response and for the mixing current at frequency $2 \omega_{1}-\omega_{2}$ from two external currents at frequencies $\omega_{1}$ and $\omega_{2} \approx 2 \omega_{1}$ due to a third order process. This pseudo-harmonic mixing probes the nonlinearity of the intervortex force and its interplay with pinning. Explicit results for the film geometry are given for the surface impedance $Z_{s}$ and the mixing current amplitude $\delta j^{(3)}$ as functions of external frequencies, crossover frequency $\omega_{c} \propto n_{i} d \omega_{D}$ (measuring the pinning), and diffusion frequency $\omega_{D}$ of free $2 \mathrm{DV}$.
\end{abstract}

Introduction The $a c$ response of type II superconductors in the mixed state has been of interest for decades [0]. The subject is complicated because of the interplay between vortex structure, GinzburgLandau-London electrodynamics, and pinning.

Recently, the high- $T_{c}$ materials which are highly anisotropic and extremely type II have stimulated new research: Anisotropic continuum theories for (dense) flux line lattices have been worked out in great detail [2].

There are new problems in the case of layered high- $T_{c}$ superconductors if these require the LawrenceDoniach approach [0]. This is the case for the most anisotropic high $-T_{c}$ materials, e.g. the $B i$ and $T l$ based superconductors. The new concept is based on "pancake" or two-dimensional vortices (2DV) with their cores residing inside the superconducting layers [4]. They form an Abrikosov vortex line by being stacked on top of each other. This leads to an enhancement of the short-range fluctuations of the flux line coordinates.

A study of $a c$ vortex dynamics based on the $2 \mathrm{DV}$ concept has been begun in [0] for the simplest case of a single Abrikosov flux line perpendicular to the layers. We have set up Fokker-Planck equations for the distribution functions of the $2 \mathrm{DV}$ coordinates and solved them approximately for the thermally averaged shifts $S_{n}(t)$ ( $n$ : layer number). The linear response to an external driving field was calculated taking account of point-like pinning centers arranged randomly along the lux line. The present work continues the program by giving a more detailed theory of the linear response using the full, i.e., untruncated electromagnetic interaction. This becomes possible by solving the Fokker-Planck equations in the adiabatic approximation when scale frequencies are small in comparison to the diffusion frequency of free 2DV. Furthermore, we study pseudoharmonic mixing as an example of a nonlinear ac response. 
Basic Equations A pancake vortex in layer $n$ at in-plane position $\rho$ is attracted by another vortex in layer $m \neq n$ and at $\boldsymbol{\rho}=\mathbf{0}$ by the electromagnetic pair force [3]

$$
\mathbf{F}_{n m}(\boldsymbol{\rho})=-a k_{B} T \frac{d}{\lambda}\left(e^{-|n-m| d / \lambda}-\exp \left(-\sqrt{|n-m|^{2} d^{2} / \lambda^{2}+\rho^{2} / \lambda^{2}}\right)\right) \mathbf{e}_{\rho} / \rho,
$$

where $a \equiv \Phi_{0}^{2} d /\left(16 \pi^{2} \epsilon(\rho) \lambda^{2} k_{B} T\right)$ is the Kosterlitz-Thouless parameter $\left(\Phi_{0}\right.$ : flux quantum, $\epsilon(\rho) \approx 1$ away from the transition). We concentrate on highly anisotropic layered superconductors (or suitable multilayers) when $\lambda \ll \lambda_{J}=d \lambda_{c} / \lambda \equiv \Gamma d$ holds. In that case, the Josephson coupling [6,7] can be neglected in comparison with the electromagnetic coupling between pancake vortices.

The electromagnetic force (1) has a range across the layers equal to the penetration length $\lambda$ which is much larger than the layer thickness $d$. By using this condition, $\lambda \gg d$, Bulaevskii, Meshkov and Feinberg [0] proposed a mean field approach in which each pancake vortex at position $(\rho, n)$ is exposed to a total force

$$
\mathbf{F}_{n}(\boldsymbol{\rho})=\sum_{m \neq n} \int d^{2} \boldsymbol{\rho}^{\prime} \mathbf{F}_{n m}\left(\boldsymbol{\rho}-\boldsymbol{\rho}^{\prime}\right) \boldsymbol{\Gamma}_{m}\left(\boldsymbol{\rho}^{\prime}, t\right)
$$

$\Gamma_{m}\left(\boldsymbol{\rho}^{\prime}, t\right)$ is the probability distribution function for the vortex positions. It obeys the Fokker-Planck equation

$$
\frac{\partial}{\partial t} \Gamma_{n}=-\mu \operatorname{div}_{\rho}\left\{\left[\mathbf{F}_{n}(\boldsymbol{\rho})+f_{n}(t) \mathbf{e}_{x}+\mathbf{F}_{n}^{(\operatorname{pin})}(\boldsymbol{\rho})\right] \Gamma_{n}-k_{B} T \operatorname{grad}_{\rho} \Gamma_{n}\right\} .
$$

In (3), the force $f_{n}$ in $x$-direction due to a transport current $j_{n}$ in $y$-direction is

$$
f_{n}(t)=d \frac{\Phi_{0}}{c}\left[j_{y}(t) \frac{\cosh n d / \lambda}{\cosh w / \lambda}\right] \Theta(w-|n d|),
$$

and a pinning force $\mathbf{F}_{n}^{(\text {pin) }}$ has also been added. Note that $\mathbf{F}_{n}$ also takes care of the currents created by deformations of the vortex line. We consider a film of thickness $2 w \gg \lambda$. The mobility of the pancake vortices is related to the flux flow resistivity $\rho_{f}$ by $\mu=\rho_{f} c^{2} /\left(B d \Phi_{0}\right)$.

The vortex shifts which are in $x$-direction follow from

$$
\mathbf{S}_{n}(t)=\int d^{2} \rho \rho \Gamma_{n}(\rho, t)=S_{n}(t) \mathbf{e}_{x}
$$

and contribute to the electric field in $y$-direction

$$
E_{n}=\frac{4 \pi \lambda^{2}}{c^{2}} \frac{\partial j_{n}}{\partial t}+\frac{1}{c} B \frac{d S_{n}}{d t}
$$

Our task is to evaluate $S_{n}$ as function of the driving current $j_{y}(t)$.

It is very difficult to solve (2) and (3). We therefore present an approximate treatment valid under conditions: i) $\omega \ll \omega_{D}$ and ii) $\omega_{c} \ll \omega_{D}$. The first condition requires the relevant frequencies in $j(t)$ to be much smaller than the diffusion frequency $\omega_{D} \equiv \mu a k_{B} T /\left(2 \lambda^{2}\right)$. Condition ii) is a restriction on the number of pinning sites $n_{i}$ per length which defines a scale frequency $\omega_{c}=O\left(n_{i} d \omega_{D}\right)$. As shown earlier [5], the distribution function $\Gamma_{n}$ is approximated by

$$
\Gamma_{n}(\boldsymbol{\rho}, t)=\Gamma_{e n}\left(\boldsymbol{\rho}-\mathbf{S}_{n}(t)\right),
$$

where $\Gamma_{e n}$ is the equilibrium distribution function, i.e. the solution of $(3)$ for $f_{n} \equiv 0$. The corrections to (7) are of the order of $\kappa^{2} \lambda^{2}=O\left(\left(\omega_{c}-i \omega\right) / \omega_{D}\right) \ll 1$, where $\kappa^{-1}$ is the actual penetration length of vortex perturbations from the surface into the superconducting film (note that $w \gg \lambda$ ). We also found that $\Gamma_{e n}$ is well approximated by a Gaussian distribution $\Gamma_{e n}(\rho)=\left(\gamma_{n}^{2} / \pi\right) \exp \left(-\gamma_{n}^{2} \rho^{2}\right)$, provided that $\left\langle\rho^{2}\right\rangle_{\Gamma_{e n}} \ll \lambda^{2}$ holds and $\gamma_{n}^{2}=\left\langle\rho^{2}\right\rangle_{\Gamma_{e n}}^{-1}$ is determined self-consistently. A bracket $\langle\ldots\rangle_{\Gamma_{e}}$ means an average with respect to the equilibrium distribution function $\Gamma_{e}$. The last inequality requires temperatures below the $K T$-transition such that $a \ln a \gg 1$.

Inserting (7) into (3), using equation (5), and performing $\int d^{2} \rho$ on the resulting equation the relation

$$
\frac{1}{\mu} \frac{d \mathbf{S}_{n}}{d t}-f_{n}(t) \mathbf{e}_{x}-\delta_{n p}\left\langle\mathbf{F}^{(\text {pin })}\left(\boldsymbol{\rho}+\mathbf{S}_{n}\right)\right\rangle_{\Gamma_{e n}}-\left\langle\left\langle\sum_{m \neq m} \mathbf{F}_{n m}\left(\boldsymbol{\rho}-\boldsymbol{\rho}^{\prime}+\mathbf{S}_{n}-\mathbf{S}_{m}\right)\right\rangle\right\rangle_{\Gamma_{e n} \Gamma_{e m}}=\mathbf{0}
$$


is obtained. It is central to the following calculations. In (8), equivalent pins are assumed and $\delta_{n p}$ takes account of layers $p$ containing a pin. Eventually, a sum over pins will be performed.

Using (8), we develop two coupled nonlinear equations for free and pinned vortex shifts in a continuous approximation if $S_{n} \rightarrow S(\zeta=z / \lambda, t)$. Free vortex shifts are denoted as $S$. Doing a gradient expansion and retaining dominant terms up to the third order, the equation for the slowly varying part in $S(\zeta)$ is obtained:

$$
\frac{1}{\mu} \frac{\partial S}{\partial t}+\nu S\left[\frac{A K}{A+K}+\frac{A_{2} K^{4}}{(A+K)^{4}} S^{2}\right]-\frac{\partial^{2} S}{\partial \zeta^{2}}\left[C+C_{2}\left(\frac{\partial S}{\partial \zeta}\right)^{2}\right]=f(t) \delta(|\zeta|-w / \lambda) .
$$

According to (4), the quantity $f(t)$ in (9) abbreviates $\Phi_{0} j_{y}(t) d / c$. In view of the condition $\kappa \lambda \ll 1$ and the slow variation of $S$ with coordinate $z$, the external current profile and consequently that of the driving forces is expressed by $\delta$-functions.

The coefficients $A, A_{2}, C$, and $C_{2}$ are found to be

$$
\begin{aligned}
A & =\frac{a k_{B} T}{2 \lambda^{2}} \ln \left\{\frac{4 \lambda^{2}}{\left(\gamma_{f}^{-2}+\gamma_{p}^{-2}\right) e^{\varepsilon}}\right\} \equiv C L, \quad C=\frac{a k_{B} T}{2 \lambda^{2}}, \\
A_{2} & =\frac{a k_{B} T}{16 \lambda^{4}} \ln \left\{\frac{4 \lambda^{2}}{\left(\gamma_{f}^{-2}+\gamma_{p}^{-2}\right) e^{(7 \varepsilon-9)}}\right\} \equiv C \frac{L_{1}}{8 \lambda^{2}}, C_{2}=-\frac{9}{4 \lambda^{2}} C,
\end{aligned}
$$

where $\mathcal{E}$ denotes Euler's constant.

The coefficient $A$ measures the force constant, i.e., the linear part in the self-consistent restoring force on a free vortex. As in $[0]$, this leads to the identification $\gamma_{f}^{2}=A /\left(2 k_{B} T\right)$. In practical situations, the pins bind strongly with pinning energy $U_{p}=O\left(a k_{B} T\right)[8,9]$ and a corresponding spring constant $K \approx a k_{B} T / \xi_{a b}^{2} \gg A$. We neglect thermally activated depinning of pinned pancake vortices and take $\mathbf{F}^{(\mathrm{pin})}=-K_{\boldsymbol{\rho}}$ for all $\boldsymbol{\rho}$. The analogue of $\gamma_{f}^{2}$ for the pinned vortices is $\gamma_{p}^{2}=(A+K) /\left(2 k_{B} T\right) \gg \gamma_{f}^{2}$. Since the shifts $S_{p}$ of pinned vortices are smaller than those of free vortices, $S_{p}$ has been eliminated perturbatively.

Finally, equation (9) must be supplemented by a boundary condition. By summing (8) over free $2 \mathrm{DV}$ and replacing summation by integration, the desired condition for the free shifts $S(z)$ can be cast into the form

$$
\lim _{\zeta \rightarrow w / \lambda^{-}}\left\{(\partial S / \partial \zeta)\left[C+(1 / 3) C_{2}(\partial S / \partial \zeta)^{2}\right]\right\}=f(t) .
$$

Linear Response The linear response to a driving force $f(t)=f_{0} \exp (-i \omega t)$ is easily found by linearization of (9) and fouriertransforming the remaining parts. Solving the equation by using the linearized boundary condition (11), one finds inside the film

$$
S(z)=\frac{f_{0} \cosh \kappa z}{C \kappa \lambda \sinh \kappa w}
$$

with

$$
[\kappa(\omega)]^{2}=\frac{1}{\lambda^{2}} \frac{\omega_{c}}{\omega_{D}}\left(1-\frac{i \omega}{\omega_{c}}\right) .
$$

The diffusion frequency is expressed as $\omega_{D}=\mu C$. Pinning is described by the frequency $\omega_{c}=n: d \frac{K L}{A+K} \omega_{D} \ll$ $\omega_{D}$. The surface impedance is thus given by

$$
Z_{\mathrm{S}}(\omega)=\frac{\rho_{f}}{1-\frac{\omega_{c}}{i \omega}} \frac{\kappa(\omega)}{\tanh \kappa(\omega) w}-i \omega \frac{4 \pi \lambda}{c^{2}}
$$

This generalizes our former result (cf. eq. (32) of [0]) where a simplified interaction model was used.

Pseudoharmonic Mixing Equation (9) will be used to evaluate the nonlinear response of the 2DV system to a transport current

$$
j_{y}(\zeta, t)=\delta(|\zeta|-w / \lambda)\left\{j_{1} \exp \left(-i \omega_{1} t+i \phi_{1}\right)+j_{2} \exp \left(-i \omega_{2} t+i \phi_{2}\right)+c . c .\right\}
$$

perturbatively.

We start from the linear shifts (12) with $f_{0} \rightarrow f_{1,2}=e^{i \phi_{1,2}} d \Phi_{0} j_{1,2} / c$ and $\kappa \rightarrow \kappa_{1,2}=\kappa\left(\omega_{1,2}\right)$, so that $S^{(\text {lin) }}\left(\zeta, \omega_{1,2}\right)=f_{1,2} \cosh \left(\kappa_{1,2} \zeta \lambda\right) /\left(C \kappa_{1,2} \lambda \sinh \kappa_{1,2} w\right)$ and we must solve (9) for the following driving force

$$
f(t)=\left\{f_{1} e^{-i\left(\omega_{1} t-\phi_{1}\right)}+f_{2} e^{-i\left(\omega_{2} t-\phi_{2}\right)}+c . c .\right\} .
$$


In pseudoharmonic mixing, one is interested in the third order electric field $E_{\delta}^{(3)}$ at the finite frequency $\omega_{\delta} \equiv 2 \omega_{1}-\omega_{2}$ generated by the combination of linear shifts $S^{(\operatorname{lin})}\left(\zeta, \omega_{1}\right)^{2} S^{(\operatorname{lin})}\left(\zeta,-\omega_{2}\right) . E_{\delta}^{(3)}$ is associated with a shift $\delta S$ which is easily shown to satisfy the equation

$$
\left(\kappa_{\delta}^{2}-\frac{d^{2}}{d \zeta^{2}}\right) \delta S=-3 \nu \frac{\bar{A}_{2}}{C} S_{1}^{2} S_{2}+\frac{C_{2}}{C}\left\{2 \frac{\partial^{2} S_{1}}{\partial \zeta^{2}} \frac{\partial S_{1}}{\partial \zeta} \frac{\partial S_{2}}{\partial \zeta}+\frac{\partial^{2} S_{2}}{\partial \zeta^{2}}\left(\frac{\partial S_{1}}{\partial \zeta}\right)^{2}\right\}
$$

where $\bar{A}_{2}$ abbreviates $A_{2} K^{4} /(A+K)^{4}$ and $S_{1,2}$ denotes the solution (12) of the linearized equation (9) for the frequencies $\omega_{1}$ and $-\omega_{2}$, respectively. The renormalized wave number $\kappa_{\delta}$ is given by (13) with $\omega$ specialized to $\omega_{\delta}$.

The solution of (16) is obtained as the sum of a homogenous solution and a particular inhomogenous solution. Eventually, we find the mixing electric fields from (6).

We consider the limit $K \gg A$ and the cases of a thin $\left(\left|\kappa\left(\omega_{\nu}\right) \lambda\right| \ll\left|\kappa\left(\omega_{\nu}\right) w\right| \ll 1\right.$ for $\left.\nu=1,2, \pm, \delta\right)$ and a thick $\left(\left|\kappa\left(\omega_{\nu}\right) w\right| \gg 1\right)$ film. In a thin film, the electric field $E_{\delta}^{(3)}$ is homogeneous across the sample and we find for the induced electric field $E_{\delta}^{(3)}$

$$
E_{\delta}^{(3)}=3 \frac{i \omega_{\delta} B}{c} \frac{L_{1}}{L}\left(\frac{\lambda}{w}\right)^{3} \frac{f_{1}^{2} f_{2}^{*}}{\lambda^{2}\left(a k_{B} T\right)^{3} \kappa\left(\omega_{1}\right)^{4} \kappa\left(-\omega_{2}\right)^{2}} .
$$

While $E_{\delta}^{(3)}$ is homogeneous across the (thin) film, the induced current density $j_{\delta}^{(3)}$ is not. It is confined to surface layers of thickness $\lambda$. We thus relate $E_{\delta}^{(3)}$ to the total current amplitude $I_{\delta}^{(3)}$ in the film using (14) in the appropriate limit $\left|\kappa_{\delta} w\right| \ll 1: E_{\delta}^{(3)}=-i \omega_{\delta} \rho_{f} /\left(\omega_{c}-i \omega_{\delta}\right) \mathrm{I}_{\delta}^{(3)} /(2 w)$. I $\mathrm{I}_{\delta}$ is related to the current density amplitude on one surface by $\mathrm{I}_{\delta}^{(3)}=2 \lambda j_{\delta}^{(3)}$. Introducing the scale for the current density $j_{0}=$ $\Phi_{0} c /\left(2 \lambda(4 \pi \lambda)^{2}\right)$, which is about the current density generated by a vortex at a distance $\lambda$ from its core, we finally get the thin film formula

$$
j_{\delta}^{(3)}=-\frac{3}{8} \frac{L_{1}}{L} \frac{j_{1}^{2} j_{2}}{j_{0}^{2}} \exp \left[i\left(2 \phi_{1}-\phi_{2}\right)\right]\left(\frac{\lambda}{w}\right)^{2} \frac{\omega_{c} \omega_{D}^{2}}{\left(\omega_{c}-i \omega_{1}\right)^{2}\left(\omega_{c}+i \omega_{2}\right)} .
$$

The opposite limit of a thick film, $\left|\kappa_{\nu} w\right| \gg 1, \nu=1,2, \pm, \delta$, can also be considered: The formula corresponding to (18) then reads

$$
j_{\delta}^{(3)}=-\frac{3}{8} \frac{L_{1}}{L} \frac{j_{1}^{2} j_{2}}{j_{0}^{2}} \exp \left[i\left(2 \phi_{1}-\phi_{2}\right)\right] \frac{\omega_{c} \omega_{D}-6\left(\omega_{c}-i \omega_{1}\right) \sqrt{\omega_{c}+i \omega_{2}} \sqrt{\omega_{c}-i \omega_{\delta}} L / L_{1}}{\left(\omega_{c}-i \omega_{1}\right) \sqrt{\omega_{c}+i \omega_{2}}\left[2 \sqrt{\omega_{c}-i \omega_{1}}+\sqrt{\omega_{c}+i \omega_{2}}+\sqrt{\omega_{c}-i \omega_{\delta}}\right]} .
$$

\section{References}

[1] Gorkov, L.P., Kopnin, N.B., Usp. Fiz. Nauk 116 (1975) 413.

[2] Koshelev, A.E., Vinokur, V.M, Physica C 173 (1991) 465; Coffey, M.W., Clem, J.R., Phys. Rev. Lett. 67 (1991) 386; Phys. Rev. B 45 (1992) 9872; Phys. Rev. B 45 (1992) 11757; Brandt, E.H., Phys. Rev. Lett. 67 (1991) 2219; Z. Phys. 80 (1990) 167; Physica C 195 (1992) 1; Sonin, E.B., Tagantsev, A.K., Traito, K.B., Phys. Rev. B 46 (1992) 5830.

[3] Lawrence, W.E., Doniach, S. in: Proc. 12th Int. Conf. Low Temp. Phys. LT 12, ed. E. Kanda (Academic Press of Japan, Kyoto 1971) p 361.

[4] Feigel'man, M.V., Geshkenbein, V.B., Larkin, A.I., Physica C 167 (1990) 177; Clem, J.R., Bull. Am. Phys. Soc. 35 (1990) 260; Phys. Rev. B 43 (1991) 7837; Artemenko, S.N., Kruglov, A.N., Phys. Lett. A 143 (1990) 485; Physica C 173 (1991) 125; Guinea, F., Phys. Rev. B 42 (1990) 6244; Buzdin, A., Feinberg, D., J. Phys. France 51 (1990) 1971; Bulaevskii, L.N., Meshkov, S.V., Feinberg, D., Phys. Rev. B 43 (1991) 3728.

[5] Artemenko, S.N., Wonneberger, W., Physica C 192 (1992) 453.

[6] Bulaevskii, L.N., Ledvij, M., Kogan, V.G., Phys. Rev. B 46 (1992) 11807.

[7] Fischer, K.H., Physica C 193 (1992) 401.

[8] Brandt, E.H., Europhys. Lett. 18 (1992) 635.

[9] Gerhäuser, W., Ries, G., Neumüller, H.W., Schmidt, W., Eibl, O., Saemann-Ischenko, G., Klaumünzer, S., Phys. Rev. Lett. 68 (1992) 879. 